\begin{tabular}{|l|l|}
\hline Sta 15 & \multicolumn{1}{|c|}{ Pago 1 of $\frac{1}{1}$} \\
\hline
\end{tabular}

\begin{tabular}{|c|c|}
\hline $\begin{array}{l}\text { 2. To: (Receiving Organization) } \\
\text { Distribution }\end{array}$ & $\begin{array}{l}\text { 3. From: (Originating Organization) } \\
\text { Special Projects }\end{array}$ \\
\hline $\begin{array}{l}\text { 5. Proj./Prog./Dept./Div.: } 10,459 / 4 \mathrm{~J} 60 \\
\text { Accel Deact Proj/River Corr Proj }\end{array}$ & $\begin{array}{l}\text { 6. Design Authority/Design Agen } \\
\text { E. N. Dodd, III }\end{array}$ \\
\hline
\end{tabular}

8. Originator Remarks:

For approval and release.

11. Receiver Remarks:

11A. Design Baseline Document? $\square$ Yes $\bigotimes$ No

\author{
4. Related EDT No.: \\ $\mathrm{N} / \mathrm{A}$ \\ 7. Purchase Order No: \\ $\mathrm{N} / \mathrm{A}$ \\ 9. Equip./Component No.: \\ $\mathrm{N} / \mathrm{A}$ \\ 10. System/Bldg./Facility: \\ $\mathrm{N} / \mathrm{A}$ \\ 12. Major Assm. Dwg. No.:
}

N/A

13. Permit/Permit Application No.:

$\mathrm{N} / \mathrm{A}$

14. Required Response Date:

$\mathrm{N} / \mathrm{A}$

\begin{tabular}{|c|c|c|c|c|c|c|c|c|}
\hline 15. & & DATA TR & NSMITTE & & (F) & (G) & (H) & (I) \\
\hline $\begin{array}{l}\text { (A) } \\
\text { Item } \\
\text { No. }\end{array}$ & (B) Document/Drawing No. & $\begin{array}{l}\text { (C) Sheet } \\
\text { No. }\end{array}$ & $\begin{array}{l}\text { (D) Rev. } \\
\text { No. }\end{array}$ & (E) Title or Description of Data Transmitted & $\begin{array}{l}\text { Approval } \\
\text { Desig- } \\
\text { nator }\end{array}$ & $\begin{array}{c}\text { Reason } \\
\text { for Trans- } \\
\text { mittal }\end{array}$ & $\begin{array}{c}\text { Origi- } \\
\text { nator } \\
\text { Dispo- } \\
\text { stion }\end{array}$ & $\begin{array}{c}\text { Receiv- } \\
\text { er } \\
\text { Dispo- } \\
\text { sition }\end{array}$ \\
\hline 1 & $\mathrm{HNE}-6071$ & & 0 & 200 Area Deactivation & $S Q$ & 1 & & \\
\hline & & & & Project Facilities & & & & \\
\hline & & & & Authorization Envelope & & & & \\
\hline & & & & Document & & & & \\
\hline & & & & & & & & \\
\hline & & & & & & & & \\
\hline & & & & & & & & \\
\hline
\end{tabular}

16.

KEY

\begin{tabular}{|c|l|}
\hline Approval Designator (F) & \\
\hline $\begin{array}{c}\text { E, S, Q, D OR N/A } \\
\text { (See WHC-CM-3-5, } \\
\text { Sec. 12.7) }\end{array}$ & $\begin{array}{l}\text { 1. Approval } \\
\text { 2. Release } \\
\text { 3. Information }\end{array}$ \\
\hline
\end{tabular}

Reason for Transmittal (G)

4. Review

5. Post-Review

6. Dist. (Receipt Acknow. Required)
Disposition $(\mathrm{H}) \&(\mathrm{l})$

$\begin{array}{ll}\text { 1. Approved } & \text { 4. Reviewed no/comment } \\ \text { 2. Approved w/comment } & \text { 5. Reviewed w/comment } \\ \text { 3. Disapproved w/comment } & \text { 6. Receipt acknowledged }\end{array}$
SIGNATURE/DISTRIBUTION

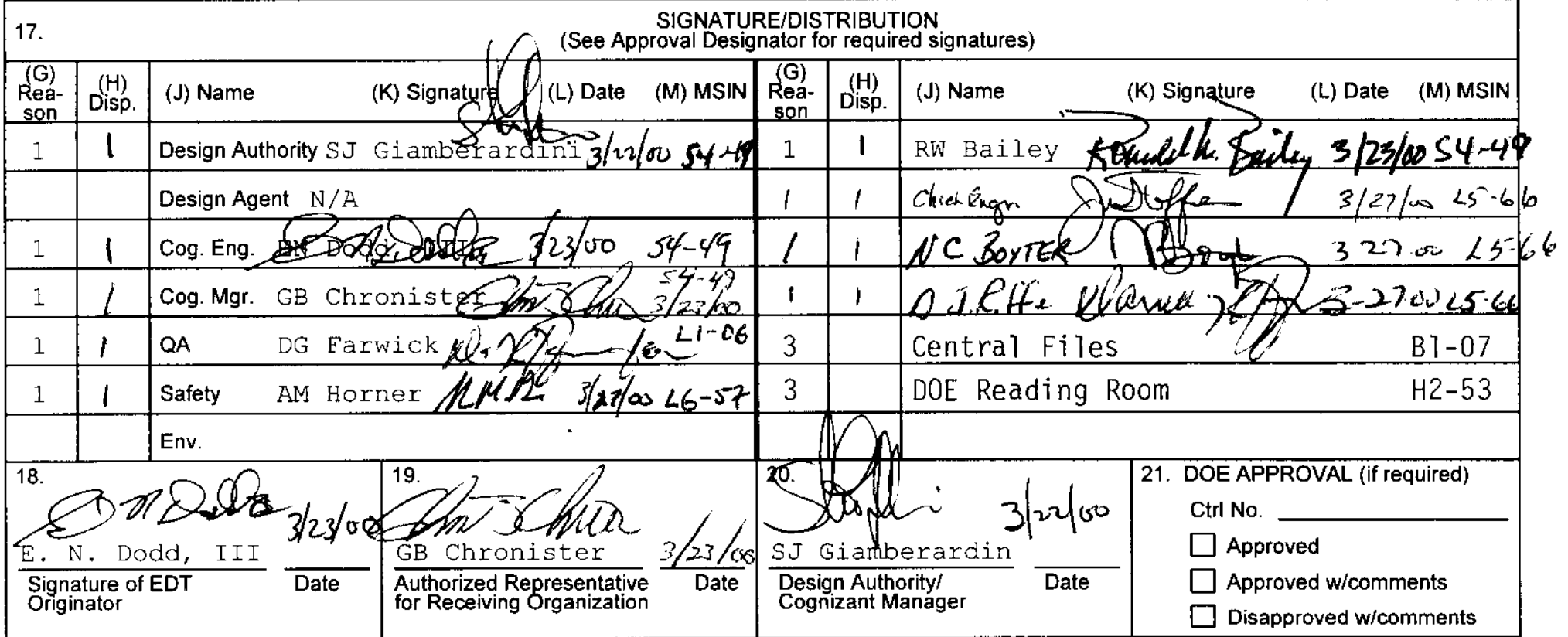


S

HNF-6071

Revision 0

\section{Area Deactivation Project Facilities \\ Authorization Envelope Document}

Prepared for the U.S. Department of Energy

Assistant Secretary for Environmental Management

Project Hanford Management Contractor for the

U.S. Department of Energy under Contract DE-AC06-96RL13200

Fluor Hanford

P.O. Box 1000

Richland, Washington 
HNF-6071

Revision 0

EDT 610387

\title{
200 Area Deactivation Project Facilities Authorization Envelope Document
}

\author{
E. N. Dodd III \\ Fluor Hanford, Inc
}

\section{Date Published}

March 2000

Prepared for the U.S. Department of Energy

Assistant Secretary for Environmental Management

Project Hanford Management Contractor for the

U.S. Department of Energy under Contract DE-AC06-96RL13200

Fluor Hanford

P.O. Box 1000

Richland, Washington
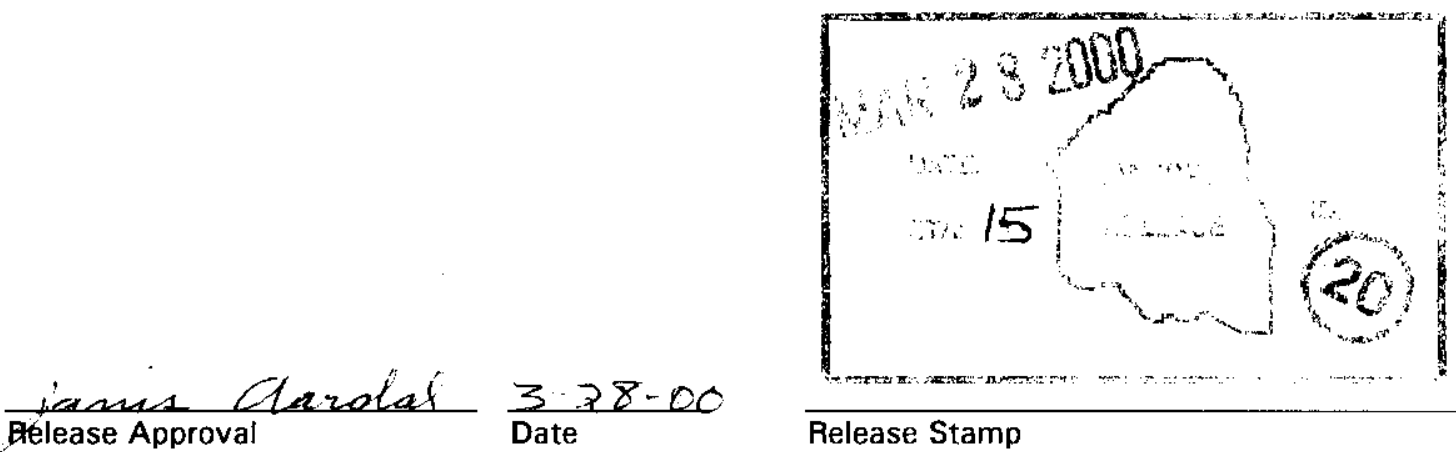

Release Stamp 
HNF-6071, Rev. 0

Key Words: Authorization Envelopes, 200 Area Deactivation Project, Authorization Agreement, AA, PUREX Tunnels, 224-T Facility, Category 2 Nuclear Facilities

\begin{abstract}
This document identifies the authorization envelopes for the 200 Area Deactivation Project facilities as required by HNF-PRO-2701, Authorization Envelope and Authorization Agreement. The Authorization Agreements (AA's) do not identify the specific set of environmental safety and health requirements that are applicable to the facility. Therefore, the facility Authorization Envelopes are defined here to identify the applicable requirements.
\end{abstract}


TRADEMARK DISCLAIMER

Reference herein to any specific commercial product, process,

or service by trade name, trademark, manufacturer, or

otherwise, does not necessarily constitute or imply its

endorsement, recommendation, or favoring by the United

States Government or any agency thereof or its contractors or subcontractors.

This report has been reproduced from the best available copy.

Printed in the United States of America

Total Pages: 
HNF-6071, Rev. 0

\section{Area Deactivation Project Facilities Authorization Envelope Document}

\subsection{Purpose}

This document identifies the authorization envelopes for the 200 Area Deactivation Project facilities as required by HNF-PRO-2701, Authorization Envelope and Authorization Agreement.

This document includes the PUREX Tunnels and the 224-T facility, both of which are category 2 nuclear facilities. While both facilities have a authorization agreement (AA) as required by HNF-PRO-2701, the AA's do not identify the specific set of environmental safety and health requirements that are applicable to the facility. Therefore, the facility Authorization Envelopes are defined here to identify the applicable requirements.

\subsection{Authorization Envelopes}

The authorization envelopes are defined in the matrices below. In addition, Appendix A of this document includes the basis for exclusion for requirements documents not included in the facility authorization envelopes. The authorization envelopes will be revised at least annually. 


\begin{tabular}{|c|c|}
\hline \multicolumn{2}{|r|}{ 222-U \& 222-T, Industrial Facility, FH } \\
\hline Safety Basis & HNF-4538, 222-T and 222-U Surveillance and Maintenance Hazards Evaluation \\
\hline $\begin{array}{l}\text { ES\&H Requirements Basis } \\
\text { - the requirements identified } \\
\text { here are taken from the } \\
\text { PHMC, Part III, Section J, } \\
\text { Appendix C, and reflect } \\
\text { only those orders that are } \\
\text { applicable and implemented } \\
\text { by the facility organization. }\end{array}$ & 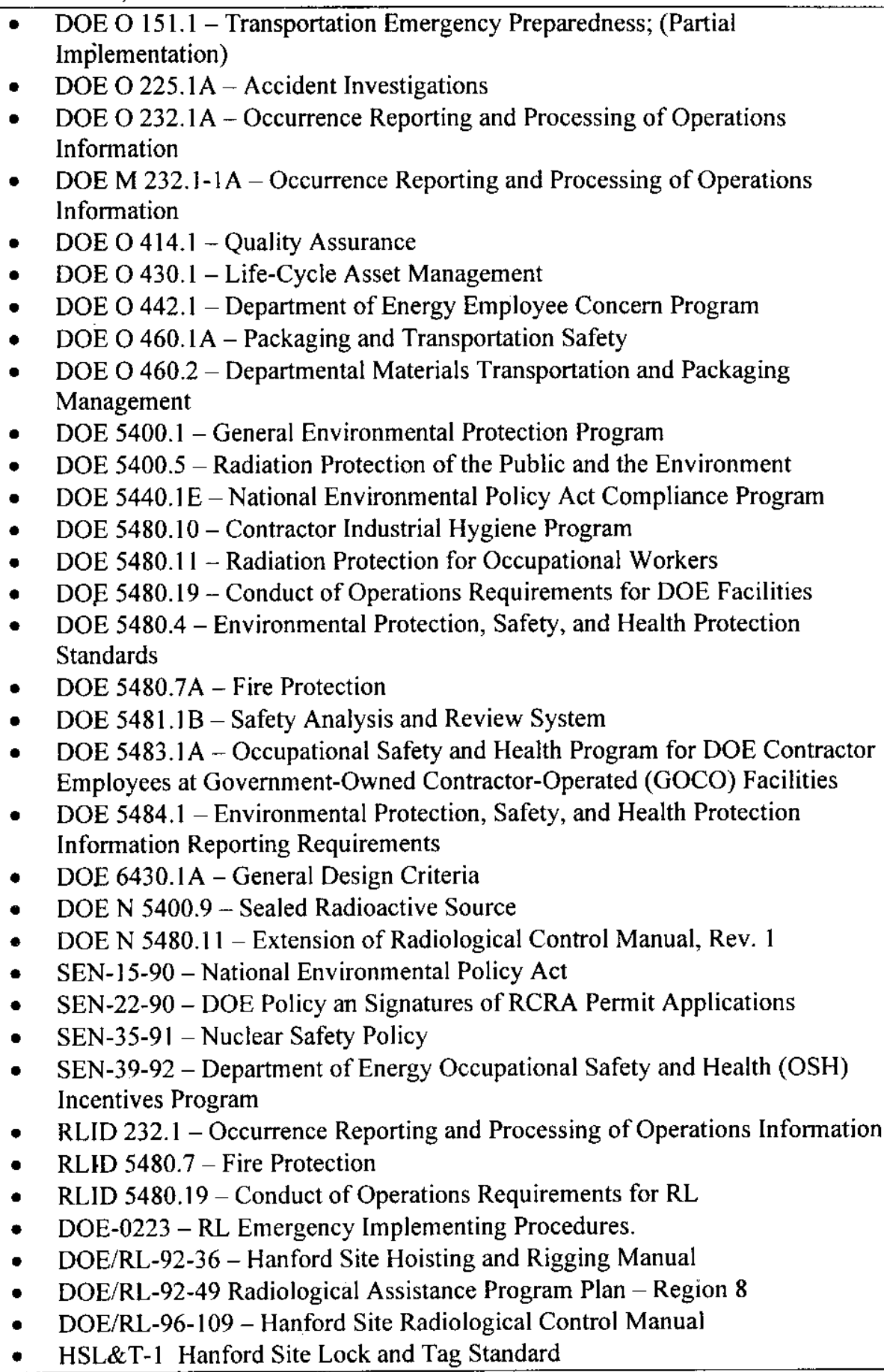 \\
\hline $\begin{array}{l}\text { Environmental Protection } \\
\text { Basis }\end{array}$ & None Identified \\
\hline
\end{tabular}




\begin{tabular}{|c|c|}
\hline \multicolumn{2}{|r|}{ 242-B/BL, Radiological Facility, FH } \\
\hline Safety Basis & HNF-3582, Auditable Safety Analysis for 242B/BL Facility, Jan. 13, 1999. \\
\hline $\begin{array}{l}\text { ES\&H Requirements Basis } \\
\text { - the requirements identified } \\
\text { here are taken from the } \\
\text { PHMC, Part III, Section J, } \\
\text { Appendix C, and reflect } \\
\text { only those orders that are } \\
\text { applicable and implemented } \\
\text { by the facility organization. }\end{array}$ & 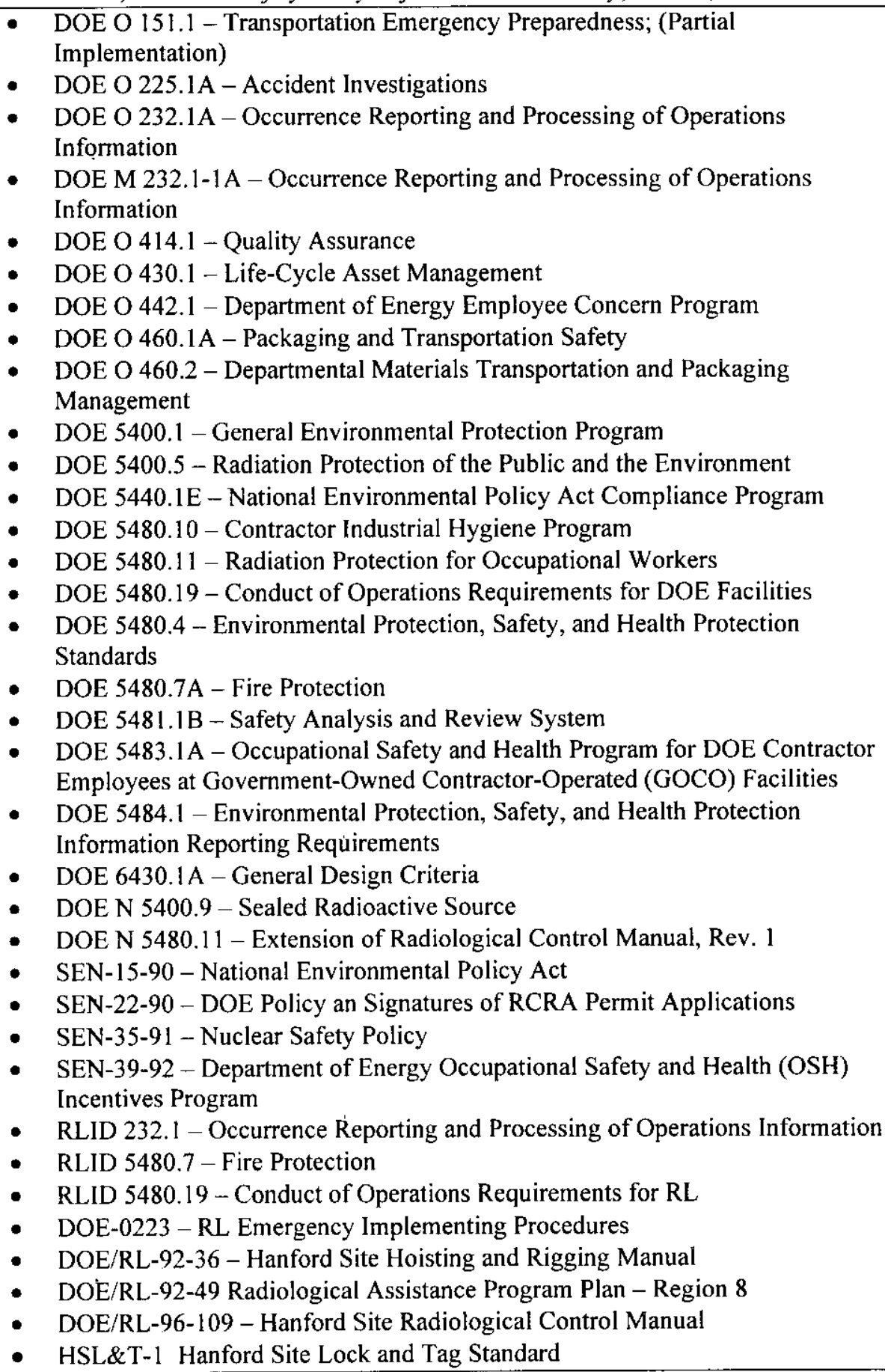 \\
\hline $\begin{array}{l}\text { Environmental Protection } \\
\text { Basis }\end{array}$ & None are identified. \\
\hline
\end{tabular}




\begin{tabular}{|c|c|}
\hline \multicolumn{2}{|r|}{ 231-Z, Category 3 Nuclear Facility, FH } \\
\hline Safety Basis & HNF-4022, $231-Z$ Safety Analysis and Documentation for Characterization \\
\hline $\begin{array}{l}\text { ES\&H Requirements Basis } \\
\text { - the requirements identified } \\
\text { here are taken from the } \\
\text { PHMC, Part III, Section J, } \\
\text { Appendix C, and reflect } \\
\text { only those orders that are } \\
\text { applicable and implemented } \\
\text { by the facility organization. }\end{array}$ & 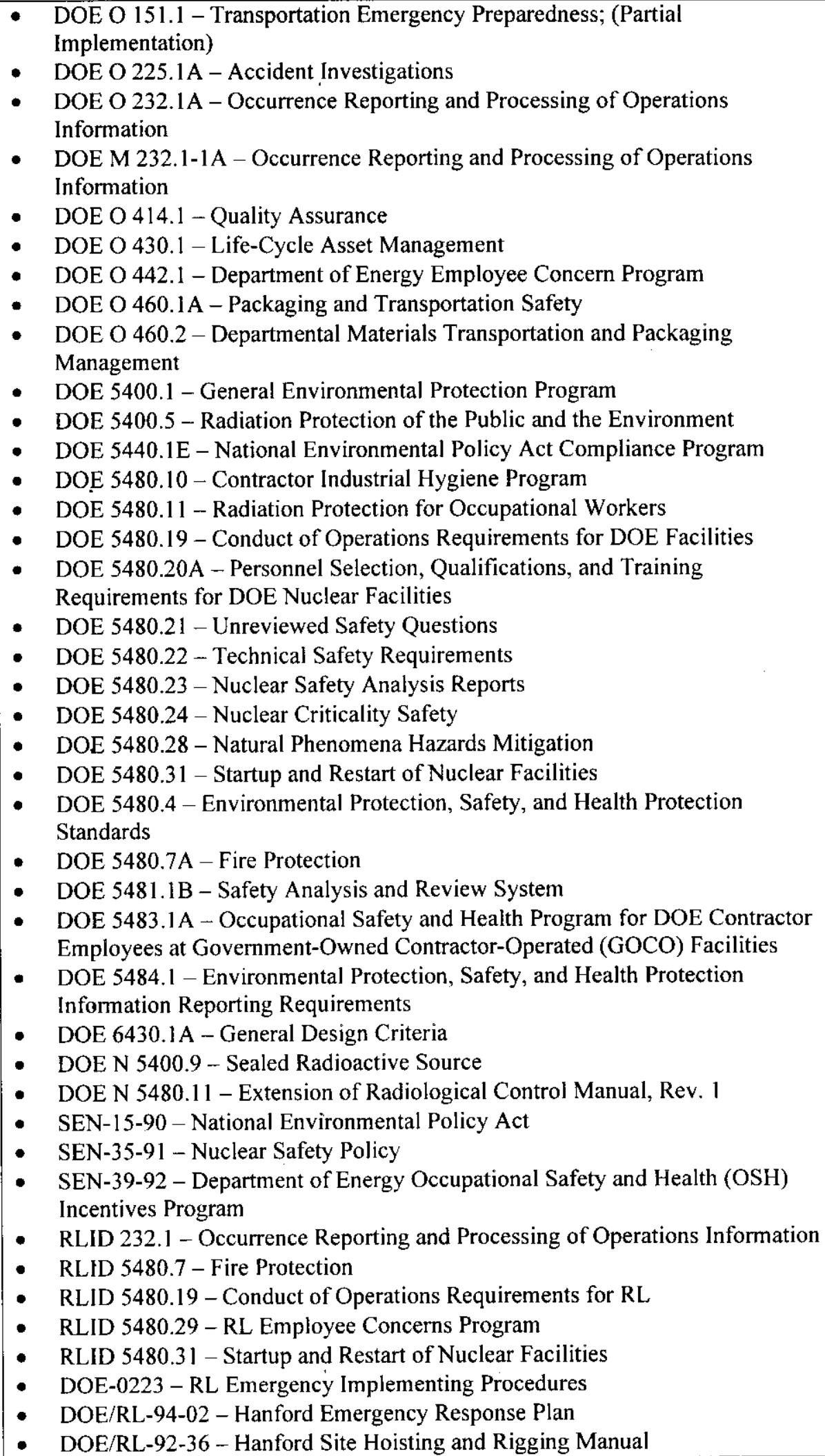 \\
\hline
\end{tabular}


HNF-6071, Rev. 0

231-Z, Category 3 Nuclear Facility, FH

\begin{tabular}{|c|c|}
\hline \multicolumn{2}{|r|}{ 231-Z, Category 3 Nuclear Facility, FH } \\
\hline & $\begin{array}{l}\text { - DOE/RL-92-49 Radiological Assistance Program Plan - Region } 8 \\
\text { - } \quad \text { DOE/RL-96-109 - Hanford Site Radiological Control Manual } \\
\text { - HSL\&T-1 - Hanford Site Lock and Tag Standard } \\
\text { - DOE-0225 - Hanford Emergency Assessment Resource Manual (HEARM) }\end{array}$ \\
\hline $\begin{array}{l}\text { Environmental Protection } \\
\text { Basis }\end{array}$ & Monthly radiological survey of facility exterior. Documented in email. \\
\hline
\end{tabular}




\begin{tabular}{|c|c|}
\hline \multicolumn{2}{|r|}{ 224-T, Category 2 Nuclear Facility, FH } \\
\hline Safety Basis & $\begin{array}{l}\text { WHC-SD-WM-ISB-004, Rev. 1, Transuranic Storage and Assay Facility } \\
\text { Interim Safety Basis, February 1997. } \\
\text { WHC-SD-WM-ISB-004, Rev. 1, Transuranic Storage and Assay Facility } \\
\text { Interim Safety Basis, Appendix E, "Safety Evaluation Report." } \\
\text { WHC-SD-WM-TSR-002, Rev. 0, TRUSAF Interim Operational Safety } \\
\text { Requirements Document, February } 1997 . \\
\text { WHC-SD-WM-FHA-007, Fire Hazards Analysis of Transuranic Waste Storage } \\
\text { and Assay Facility. }\end{array}$ \\
\hline $\begin{array}{l}\text { ES\&H Requirements Basis } \\
\text { - the requirements identified } \\
\text { here are taken from the } \\
\text { PHMC, Part III, Section J, } \\
\text { Appendix C, and reflect } \\
\text { only those orders that are } \\
\text { applicable and implemented } \\
\text { by the facility organization. }\end{array}$ & 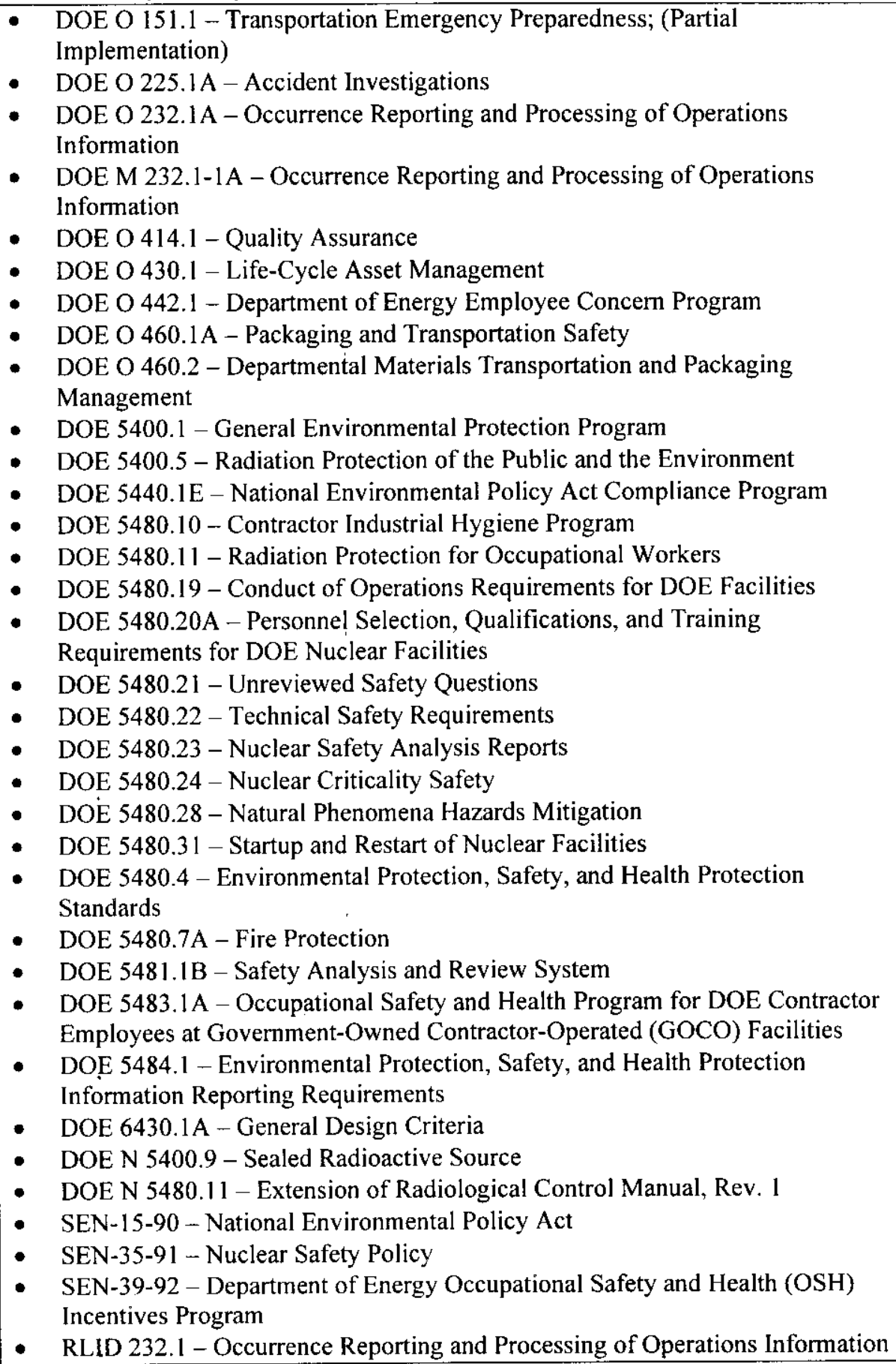 \\
\hline
\end{tabular}


HNF-6071, Rev. 0

\section{4-T, Category 2 Nuclear Facility, FH}

\begin{tabular}{|c|c|}
\hline \multicolumn{2}{|r|}{ 224-T, Category 2 Nuclear Facility, FH } \\
\hline & $\begin{array}{ll}\text { - } & \text { RLID 5480.7 - Fire Protection } \\
\text { - } & \text { RLID 5480.19 - Conduct of Operations Requirements for RL } \\
\text { - } & \text { RLID 5480.29 - RL Employee Concerns Program } \\
\text { - } & \text { RLID 5480.31 - Startup and Restart of Nuclear Facilities } \\
\text { - } & \text { DOE-0223 - RL Emergency Implementing Procedures } \\
\text { - } & \text { DOE/RL-94-02 - Hanford Emergency Response Plan } \\
\text { - } & \text { DOE/RL-92-36 - Hanford Site Hoisting and Rigging Manual } \\
\text { - } & \text { DOE/RL-92-49 Radiological Assistance Program Plan - Region } 8 \\
\text { - } & \text { DOE/RL-96-109 - Hanford Site Radiological Control Manual } \\
\text { - } & \text { DOL\&T-1 - Hanford Site Lock and Tag Standard } \\
\end{array}$ \\
\hline $\begin{array}{l}\text { Environmental Protection } \\
\text { Basis }\end{array}$ & $\begin{array}{l}\text { - DOE/RL-88-21, Hanford Dangerous Waste Part A Permit Application } \\
\text { - ERDA-1538, Waste Management Operations, Hanford Reservation, Richland, } \\
\text { Washington, December } 1975 \\
\text { - DOE/EIS-0133, Final Environmental Impact Statement, Disposal of Hanford } \\
\text { Defense High-Level Transuranic and Tank Wastes, Hanford Site, Richland, } \\
\text { Washington, December } 1987 \\
\text { Washington State Department of Health Radioactive Air Emissions Permit, FF- } \\
\text { 01, August 1993. }\end{array}$ \\
\hline
\end{tabular}




\begin{tabular}{|c|c|}
\hline \multicolumn{2}{|r|}{ PUREX Storage Tunnels, Category 2 Nuclear Facility, FH } \\
\hline Safety Basis & $\begin{array}{l}\text { - SD-HS-SAR-001, Revision 5a, PUREX Plant Final Safety Analysis Report, } \\
\text { Section 7.7, "Solid Waste,",January } 1996 . \\
\text { - HNF-SD-CP-ISB-005, Revision 0, PUREX Draft ISB, pages 36-39. }\end{array}$ \\
\hline $\begin{array}{l}\text { ES\&H Requirements Basis } \\
\text { - the requirements identified } \\
\text { here are taken from the } \\
\text { PHMC, Part III, Section J, } \\
\text { Appendix C, and reflect } \\
\text { only those orders that are } \\
\text { applicable and implemented } \\
\text { by the facility organization. }\end{array}$ & 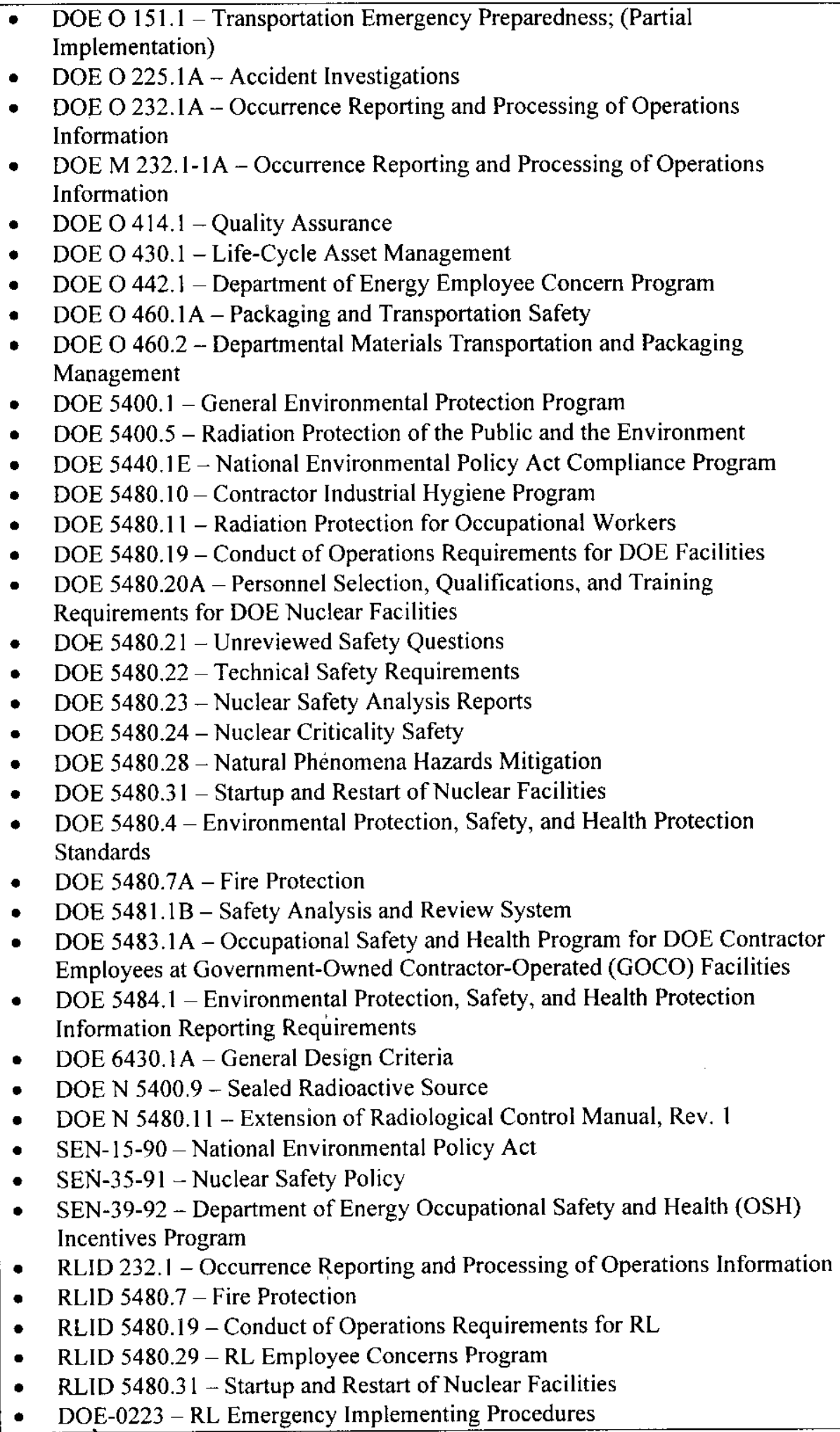 \\
\hline
\end{tabular}


HNF-6071, Rev. 0

\begin{tabular}{|c|c|}
\hline \multicolumn{2}{|r|}{ PUREX Storage Tunnels, Category 2 Nuclear Facility, FH } \\
\hline & $\begin{array}{ll}\text { - } & \text { DOE/RL-94-02 - Hanford Emergency Response Plan } \\
\text { - } & \text { DOE/RL-92-36 - Hanford Site Hoisting and Rigging Manual } \\
\text { - } & \text { DOE/RL-92-49 Radiological Assistance Program Plan - Region } 8 \\
\text { - } & \text { DOE/RL-96-109 - Hanford Site Radiological Control Manual } \\
\text { - } & \text { HSL\&T-1 - Hanford Site Lock and Tag Standard } \\
\text { - } & \text { DOE-0225 - Hanford Emergency Assessment Resource Manual (HEARM) }\end{array}$ \\
\hline $\begin{array}{l}\text { Environmental Protection } \\
\text { Basis }\end{array}$ & $\begin{array}{l}\text { - DOE/RL-90-24, Rev 4, Hanford Facility Dangerous Waste Permit Application, } \\
\text { PUREX Storage Tunnels } \\
\text { - DOE/RL-99-67, Radioactive Air Emissions Notice of Construction for } \\
\text { Reactivation of the PUREX storage Tunnel Number } 2 \\
\text { - DOE/RL-88-21, Hanford Dangerous Waste Part A Permit Application } \\
\text { - DOE/EIS-0089D, Operation of PUREX and Uranium Oxide Plant Facilities } \\
\text { Draft Environmental lmpact Statement } \\
\text { DOE/EIS-0089, Addendum to Environmental Impact Statement Operation of } \\
\text { PUREX and Uranium Oxide Plant Facilities } \\
\text { - Washington State Department of Health Radioactive Air Emissions Permit, FF- } \\
\text { 01, August 1993. }\end{array}$ \\
\hline
\end{tabular}


HNF-6071, Rev. 0

APPENDIX A 


\section{DOE Order (Appendix C) Evaluation \& Basis 200 ADP Facilities}

\begin{tabular}{|c|c|c|}
\hline $\begin{array}{l}\text { DOCUMENT } \\
\text { NUMBER }\end{array}$ & TITLE & BASIS \\
\hline DOE O 130.1 & Budget Formulation Process & Not ES\&H \\
\hline DOE M 140.1-1A & $\begin{array}{l}\text { Manual for Department of Energy Interface with } \\
\text { the Defense Nuclear Facilities Safety Board }\end{array}$ & Not ES\&H \\
\hline DOE O 151.1 & $\begin{array}{l}\text { Transportation Emergency Preparedness; Partial } \\
\text { Implementation (Chapter 5, Section 2.d) }\end{array}$ & Implemented \\
\hline DOE M 200.1-1 & $\begin{array}{l}\text { Telecommunications Security Manual (Except } \\
\text { Chapter 2) }\end{array}$ & Not ES\&H. \\
\hline DOE N 205.1 & Unclassified Cyber Security Program & Not ES\&H \\
\hline DOE O 224.1 & $\begin{array}{l}\text { Contractor Performance-Based Business } \\
\text { Management Process }\end{array}$ & Not ES\&H \\
\hline DOE O 225.1A & Accident Investigations & Implemented \\
\hline DOE O 232.1A & $\begin{array}{l}\text { Occurrence Reporting and Processing of } \\
\text { Operations } \\
\text { Information }\end{array}$ & Implemented \\
\hline DOE M 232.1-1A & $\begin{array}{l}\text { Occurrence Reporting and Processing of } \\
\text { Operations Information }\end{array}$ & Implemented \\
\hline DOE O 241.1 & Scientific and Technical Information Management & Not ES\&H \\
\hline DOE O 251.1A & Directives System Order & Not ES\&H \\
\hline DOE O 251.1 & Directive System & Not ES\&H \\
\hline DOE O 311.1A & Equal Opportunity and Diversity Program & Not ES\&H \\
\hline DOE O 350.1 & $\begin{array}{l}\text { Contractor Human Resources Management } \\
\text { Program }\end{array}$ & Not ES\&H \\
\hline DOE O 413.1 & Management Control Program & Not ES\&H \\
\hline DOE O 414.1 & Quality Assurance & Implemented \\
\hline DOE O 430.1 & Life-Cycle Asset Management & Implemented \\
\hline DOE O 440.2 & Aviation & RCP does not have aircraft \\
\hline DOE M 440.2 & Aviation Manual & RCP does not have aircraft. \\
\hline DOE O 442.1 & $\begin{array}{l}\text { Department of Energy Employee Concerns } \\
\text { Program }\end{array}$ & Implemented \\
\hline DOE O 460.1A & Packaging and Transportation Safety & Implemented \\
\hline DOE 0460.2 & $\begin{array}{l}\text { Departmental Materials Transportation and } \\
\text { Packaging Management }\end{array}$ & Implemented \\
\hline DOE O 470.1 & Safeguards and Security Program & $\begin{array}{l}\text { Security services are purchased by the } \\
\text { facility and are addressed in the site } \\
\text { S/RID and therefore do not warrant } \\
\text { inclusion here. }\end{array}$ \\
\hline DOE O 470.2 & $\begin{array}{l}\text { Safeguards and Security Independent Oversight } \\
\text { Program }\end{array}$ & Not ES\&H \\
\hline DOE O 471.1 & $\begin{array}{l}\text { Identification and Protection of Unclassified } \\
\text { Controlled Nuclear Information }\end{array}$ & Not ES\&H \\
\hline DOE $0471.2 \mathrm{~A}$ & Information Security Program & Not ES\&H \\
\hline DOE M 471.2-1 & Manual for Classified Matter Protection \& Control & Not ES\&H \\
\hline DOE O 472.1B & Personnel Security Activities & $\begin{array}{l}\text { Security services are purchased by the } \\
\text { facility and are addressed in the site } \\
\text { S/RID and therefore do not warrant } \\
\text { inclusion here. }\end{array}$ \\
\hline
\end{tabular}




\begin{tabular}{|c|c|c|}
\hline $\begin{array}{l}\text { DOCUMENT } \\
\text { NUMBER }\end{array}$ & TITLE & BASIS \\
\hline DOE M 473.2-1B & Firearms Qualifications Courses Manual & $\begin{array}{l}\text { Security services are purchased by the } \\
\text { facility and are addressed in the site } \\
\text { S/RID and therefore do not warrant } \\
\text { inclusion here. }\end{array}$ \\
\hline DOE M 474.1-2 & $\begin{array}{l}\text { Nuclear Materials Management and Safeguards } \\
\text { System Reporting and Data Submission }\end{array}$ & Not ES\&H \\
\hline DOE M 475.1-1 & Identifying Classified Information & Not ES\&H \\
\hline DOE O 481.1 & $\begin{array}{l}\text { Work for Others (Non Department of Energy } \\
\text { Funded Work) }\end{array}$ & Not ES\&H \\
\hline DOE M 481.1-1 & $\begin{array}{l}\text { Reimbursable Work for Non-Federal Sponsors } \\
\text { Process Manual }\end{array}$ & Not ES\&H \\
\hline DOE O 534.1 & Accounting & Not ES\&H \\
\hline DOE $1220.1 \mathrm{~A}$ & Congressional and Intergovernmental Affairs & Not ES\&H \\
\hline DOE 1230.2 & American Indian Tribal Government Policy & Not ES\&H \\
\hline DOE 1240.2B & $\begin{array}{l}\text { Unclassified Visits and Assignments by Foreign } \\
\text { Nationals }\end{array}$ & Not ES\&H \\
\hline DOE 1270.2B & $\begin{array}{l}\text { Safeguards Agreement with the International } \\
\text { Atomic Energy Agency }\end{array}$ & Not ES\&H \\
\hline DOE $1300.2 \mathrm{~A}$ & $\begin{array}{l}\text { Department of Energy Technical Standards } \\
\text { Program }\end{array}$ & Not ES\&H \\
\hline DOE 1300.3 & Policy on the Protection of Human Subjects & $\begin{array}{l}\text { This order does not directly impact the } \\
\text { safety of the facility. }\end{array}$ \\
\hline DOE 1324.5B & $\begin{array}{c}\text { Records Management Program } \\
\end{array}$ & $\begin{array}{l}\text { This order does not directly impact the } \\
\text { safety of the facility. }\end{array}$ \\
\hline DOE 1330.1D & Computer Software Management & $\begin{array}{l}\text { This order does not directly impact the } \\
\text { safety of the facility. }\end{array}$ \\
\hline$\overline{\mathrm{DO}} \overline{\mathrm{E}} 1340.1 \mathrm{~B}$ & $\begin{array}{l}\text { Management of Public Communications } \\
\text { Publications and Scientific, Technical and } \\
\text { Engineering Publications }\end{array}$ & Not ES\&H \\
\hline DOE 1350.1 & Audiovisual and Exhibits Management & Not ES\&H \\
\hline DOE 1410.2 & Mail Management & Not ES\&H \\
\hline DOE $1450.3 \mathrm{~A}$ & $\begin{array}{l}\text { Call Control/Verification Programs and } \\
\text { Authorized Use of Government Telephone } \\
\text { Systems }\end{array}$ & Not ES\&H \\
\hline DOE 1450.4 & $\begin{array}{l}\text { Consensual Listening-In To Or Recording } \\
\text { Telephone/Radio Conversations }\end{array}$ & Not ES\&H \\
\hline DOE 1500.3 & Foreign Travel Authorizations & Not ES\&H \\
\hline DOE 1700.1 & Freedom of Information Program & Not ES\&H \\
\hline DOE $1800.1 \mathrm{~A}$ & Privacy Act & Not ES\&H \\
\hline DOE 2030.4B & $\begin{array}{l}\text { Reporting Fraud, Waste, and Abuse to the Office } \\
\text { of Inspector General }\end{array}$ & Not ES\&H \\
\hline DOE $2100.8 \mathrm{~A}$ & $\begin{array}{l}\text { Cost Accounting, Cost Recovery, \& Interagency } \\
\text { Sharing of Information Technology Facilities }\end{array}$ & Not ES\&H. \\
\hline DOE 2110.1A & Pricing of Departmental Materials and Services & Not ES\&H \\
\hline DOE 2300.1B & Audit Resolution and Follow-Up & Not ES\&H \\
\hline DOE 2320.1C & $\begin{array}{l}\text { Cooperation With the Office of the Inspector } \\
\text { General }\end{array}$ & Not ES\&H \\
\hline DOE 2320.2B & $\begin{array}{l}\text { Establishment of Departmental Position on } \\
\text { Inspector General Reports }\end{array}$ & Not ES\&H \\
\hline DOE 3900.1B & Parking _... & Not ES\&H \\
\hline DOE $4210.9 \mathrm{~A}$ & Unsolicited Proposals & Not ES\&H \\
\hline DOE 4330.2D & In-House Energy Management & Not ES\&H \\
\hline
\end{tabular}


HNF-6071, Rev. 0

\begin{tabular}{|c|c|c|}
\hline $\begin{array}{l}\text { DOCUMENT } \\
\text { NUMBER }\end{array}$ & TITLE & BASIS \\
\hline DOE 4330.4B & Maintenance Management Program & $\begin{array}{l}\text { This order is not applicable to } 200 \text { Area } \\
\text { Deactivation Project facilities based on } \\
\text { facility life-cycle and conditions. }\end{array}$ \\
\hline DOE 5300.1C & Telecommunications & Not ES\&H \\
\hline DOE 5400.1 & General Environmental Protection Program & Implemented \\
\hline DOE 5400.5 & $\begin{array}{l}\text { Radiation Protection of the Public and the } \\
\text { Environment }\end{array}$ & Implemented \\
\hline DOE 5440.1E & $\begin{array}{l}\text { National Environmental Policy Äct Compliance } \\
\text { Program }\end{array}$ & Implemented. \\
\hline DOE 5480.10 & Contractor Industrial Hygiene Program & Implemented \\
\hline DOE 5480.11 & Radiation Protection for Occupational Workers & Implemented \\
\hline DOE 5480.16A & Firearms Safety & $\begin{array}{l}\text { Security services are purchased by the } \\
\text { facility and are addressed in the site } \\
\text { S/RID and therefore do not warrant } \\
\text { inclusion here. }\end{array}$ \\
\hline DOE 5480.17 & Site Safety Representatives & Not ES\&H \\
\hline DOE 5480.19 & $\begin{array}{l}\text { Conduct of Operations Requirements for DOE } \\
\text { Facilities }\end{array}$ & Implemented. \\
\hline DOE $5480.20 \mathrm{~A}$ & $\begin{array}{l}\text { Personnel Selection, Qualifications, and Training } \\
\text { Requirements for DOE Nuclear Facilities }\end{array}$ & $\begin{array}{l}\text { Implemented for NUCLEAR not at non- } \\
\text { nuclear facilities. }\end{array}$ \\
\hline DOE 5480.21 & Unreviewed Safety Questions & $\begin{array}{l}\text { Implemented at NUCLEAR not at non- } \\
\text { nuclear facilities. }\end{array}$ \\
\hline DÖE 5480.22 & Technical Safety Requirements & $\begin{array}{l}\text { Implemented at NUCLEAR not at non- } \\
\text { nuclear facilities. }\end{array}$ \\
\hline DOE 5480.23 & Nuclear Safety Analysis Reports & $\begin{array}{l}\text { Implemented at NUCLEAR not at non- } \\
\text { nuclear facilities. }\end{array}$ \\
\hline DOE 5480.24 & Nuclear Criticality Safety & Implemented. \\
\hline DOE $\overline{5480.26}$ & $\begin{array}{l}\text { Trending and Analysis of Operations Information } \\
\text { Using Performance Indicators }\end{array}$ & $\begin{array}{l}\text { Implemented at NUCLEAR not at non- } \\
\text { nuclear facilities. }\end{array}$ \\
\hline DOE 5480.28 & Natural Phenomena Hazards Mitigation & Implemented. \\
\hline DOE 5480.30 & Nuclear Reactor Safety Design Criteria & $\begin{array}{l}\text { This order does not directly impact the } \\
\text { safety of the facility since none of the } \\
\text { facilities are reactors. }\end{array}$ \\
\hline DOE 5480.31 & Startup and Restart of Nuclear Facilities & Implemented. \\
\hline DOE 5480.4 & $\begin{array}{l}\text { Environmental Protection, Safety, and Health } \\
\text { Protection Standards }\end{array}$ & Implemented. \\
\hline DOE 5480.6 & Safety of DOE-Owned Nuclear Reactors & $\begin{array}{l}\text { This order does not directly impact the } \\
\text { safety of the facility since none of the } \\
\text { facilities are reactors. }\end{array}$ \\
\hline DOE 5480.7A & Fire Protection & Implemented. \\
\hline$\overline{D O E} 5480.8 \mathrm{~A}$ & Contractor Occupational Medical Program & $\begin{array}{l}\text { This is a purchased service and is } \\
\text { identified in the sire S/RID. }\end{array}$ \\
\hline DOE 5480.9A & $\begin{array}{l}\text { Construction Project Safety and Health } \\
\text { Management }\end{array}$ & $\begin{array}{l}\text { No construction being performed at } 200 \\
\text { Area Deactivation Project facilities. }\end{array}$ \\
\hline DOE 5481.1B & Safety Analysis and Review System & Implemented. \\
\hline DOE 5483.1A & $\begin{array}{l}\text { Occupational Safety and Health Program for DOE } \\
\text { Contractor Employees at Government-Owned } \\
\text { Contractor-Operated (GOCO) Facilities }\end{array}$ & Implemented. \\
\hline DOE 5484.1 & $\begin{array}{l}\text { Environmental Protection, Safety, and Health } \\
\text { Protection Information Reporting Requirements }\end{array}$ & Implemented \\
\hline DOE 5530.1A & Accident Response Group & $\begin{array}{l}\text { This order does not directly impact the } \\
\text { safety of the facilities. }\end{array}$ \\
\hline
\end{tabular}




\begin{tabular}{|c|c|c|}
\hline $\begin{array}{l}\text { DOCUMENT } \\
\text { NUMBER }\end{array}$ & TITLE & BASIS \\
\hline DOE 5530.2 & Nuclear Emergency Search Team & $\begin{array}{l}\text { This order does not directly impact the } \\
\text { safety of the facilities. }\end{array}$ \\
\hline DOE 5530.3 & Radiological Assistance Program & $\begin{array}{l}\text { This order does not directly impact the } \\
\text { safety of the facilities. }\end{array}$ \\
\hline DOE 5530.4 & Aerial Measuring System & $\begin{array}{l}\text { This order does not directly impact the } \\
\text { safety of the facilities. }\end{array}$ \\
\hline DÓE 5530.5 & $\begin{array}{l}\text { Federal Radiological Monitoring and Assessment } \\
\text { Center }\end{array}$ & $\begin{array}{l}\text { This order does not directly impact the } \\
\text { safety of the facilities. }\end{array}$ \\
\hline DOE $5560.1 \mathrm{~A}$ & Priorities and Allocations Program & Not ES\&H \\
\hline DOE 5610.13 & $\begin{array}{l}\text { Joint Department of Energy/Department of } \\
\text { Defense Nuclear Weapon Safety, Security, and } \\
\text { Control Program }\end{array}$ & $\begin{array}{l}\text { This order does not directly impact the } \\
\text { safety of the facilities. }\end{array}$ \\
\hline DOE 5610.14 & $\begin{array}{l}\text { Transportation Safeguards System Program } \\
\text { Operations }\end{array}$ & $\begin{array}{l}\text { Security services are purchased by the } \\
\text { facility and are addressed in the site } \\
\text { S/RID and therefore do not warrant } \\
\text { inclusion here. }\end{array}$ \\
\hline DOE 5610.2 & Control of Weapon Data & $\begin{array}{l}\text { Security services are purchased by the } \\
\text { facility and are addressed in the site } \\
\text { S/RID and therefore do not warrant } \\
\text { inclusion here. }\end{array}$ \\
\hline DOE 5632.1C & $\begin{array}{l}\text { Protection and Control of Safeguards and Security } \\
\text { Interests }\end{array}$ & $\begin{array}{l}\text { Security services are purchased by the } \\
\text { facility and are addressed in the site } \\
\text { S/RID and therefore do not warrant } \\
\text { inclusion here. }\end{array}$ \\
\hline DOE M 5632.1C-1 & $\begin{array}{l}\text { Manual for Protection and Control of Safeguards } \\
\text { and Security Interests }\end{array}$ & $\begin{array}{l}\text { Security services are purchased by the } \\
\text { facility and are addressed in the site } \\
\text { S/RID and therefore do not warrant } \\
\text { inclusion here. }\end{array}$ \\
\hline DOE 5632.7A & Protection Force Program & $\begin{array}{l}\text { Security services are purchased by the } \\
\text { facility and are addressed in the site } \\
\text { S/RID and therefore do not warrant } \\
\text { inclusion here. }\end{array}$ \\
\hline DOE 5633.3B & Control and Accountability of Nuclear Materials & Not ES\&H \\
\hline DOE M 5639.6A-1 & $\begin{array}{l}\text { Manual of Security Requirements for the } \\
\text { Classified Automated Information System Security } \\
\text { Program }\end{array}$ & Not ES\&H \\
\hline DOE 5660.1B & Management of Nuclear Materials & Not ES\&H \\
\hline DOE 5670.1A & Management and Control of Foreign Intelligence & Not ES\&H \\
\hline DOE 5670.3 & Counterintelligence Program & Not ES\&H \\
\hline DOE 5800.1A & $\begin{array}{l}\text { Research and Development Laboratory } \\
\text { Technology Transfer Program }\end{array}$ & $\begin{array}{l}\text { This order does not directly impact the } \\
\text { safety of the facilities. }\end{array}$ \\
\hline DOE $5820.2 \mathrm{~A}$ & Radioactive Waste Management & $\begin{array}{l}\text { This order does not directly impact the } \\
\text { safety of the facilities. }\end{array}$ \\
\hline DOE 6430.1A & General Design Criteria & Implemented \\
\hline DOE N 5400.9 & Sealed Radioactive Source & Implemented \\
\hline DOE N 5480.11 & Extension of Radiological Control Manual, Rev. I & Implemented \\
\hline SEN-15-90 & National Environmental Policy Act & Implemented \\
\hline SEN-22-90 & $\begin{array}{l}\text { DOE Policy an Signatures of RCRA Permit } \\
\text { Applications }\end{array}$ & Not ES\&H \\
\hline SEN-30A-92 & $\begin{array}{l}\text { Staying the Course for Technology Transfer at the } \\
\text { Department of Energy }\end{array}$ & Not ES\&H \\
\hline DOE/RW-0333P & Quality Assurance Requirements and Descriptions & İmplemented \\
\hline SEN-35-91 & Nuclear Safety Policy & Implemented \\
\hline
\end{tabular}


HNF-6071, Rev. 0

\begin{tabular}{|l|l|l|}
\hline $\begin{array}{c}\text { DOCUMENT } \\
\text { NUMBER }\end{array}$ & \multicolumn{1}{|c|}{ TITLE } & \multicolumn{1}{c|}{ BASIS } \\
\hline SEN-39-92 & $\begin{array}{l}\text { Department of Energy Occupational Safety and } \\
\text { Health (OSH) Incentives Program }\end{array}$ & Implemented \\
\hline
\end{tabular}




\begin{tabular}{|c|c|c|}
\hline \multicolumn{3}{|c|}{ RL DIRECTIVES } \\
\hline $\begin{array}{l}\text { DOC. } \\
\text { NUMBER }\end{array}$ & TITLE & BASIS \\
\hline RLID 232.1A & $\begin{array}{l}\text { Occurrence Reporting and Processing of } \\
\text { Operations Information }\end{array}$ & Implemented \\
\hline RLID 430.1 & $\begin{array}{l}\text { Systems Engineering Criteria Document } \\
\text { and Implementing Directive }\end{array}$ & $\begin{array}{l}\text { This order does not directly impact the safety of the } \\
\text { facilities. }\end{array}$ \\
\hline RLID 470.1 & $\begin{array}{l}\text { Safeguards and Security Corrective Action } \\
\text { Management System }\end{array}$ & Not ES\&H \\
\hline RLID 470.2 & $\begin{array}{l}\text { Facility Approval and Registration of } \\
\text { Activities }\end{array}$ & Not ES\&H \\
\hline RLID 471.2B & Information Security Program & Not ES\&H \\
\hline$\overline{\text { RLID 473.1 }}$ & $\begin{array}{l}\text { Protection of Safeguards and Security } \\
\text { Interests }\end{array}$ & $\begin{array}{l}\text { Security services are purchased by the facility and are } \\
\text { addressed in the site S/RID and therefore do not warrant } \\
\text { inclusion here. }\end{array}$ \\
\hline$\overline{\text { RLID } 473.2}$ & Hanford Site Access Eligibility & Not ES\&H \\
\hline $\begin{array}{l}\text { RLID } \\
1300.1 \mathrm{D}\end{array}$ & $\begin{array}{l}\text { Richland Operations Office Facility } \\
\text { Representative Program }\end{array}$ & Not ES\&H \\
\hline $\begin{array}{l}\text { RLID } \\
1360.2 \mathrm{~B}\end{array}$ & Unclassified Computer Security Program & Not ES\&H \\
\hline RLID 5000.1 & $\begin{array}{l}\text { Baseline Execution and Management } \\
\text { Process }\end{array}$ & Not ES\&H \\
\hline RLID 5000.2 & Long Range Planning Process & Not ES\&H \\
\hline RLID 5480.7 & Fire Protection & Implemented \\
\hline RLID 5480.19 & $\begin{array}{l}\text { Conduct of Operations Requirements for } \\
\text { RL }\end{array}$ & Implemented \\
\hline RLID 5480.29 & RL Employee Concerns Program & Implemented \\
\hline RLID 5480.31 & Startup and Restart of Nuclear Facilities & Implemented \\
\hline RLID 5633.3 & $\begin{array}{l}\text { Control and Accountability of Nuclear } \\
\text { Materials at RL }\end{array}$ & Not ES\&H \\
\hline RLID 5635.1 & $\begin{array}{l}\text { Special Access and Top Secret Access } \\
\text { Authorization }\end{array}$ & Not ES\&H \\
\hline $\begin{array}{l}\text { RLID } \\
5670.3 \mathrm{~A} \\
\end{array}$ & Counterintelligence Program & Not ES\&H \\
\hline RLIP 1322.1B & RL Forms Management & Not ES\&H \\
\hline RLIP 5484.1A & $\begin{array}{l}\text { Environmental Protection, Safety, and } \\
\text { Health Protection Information Reporting } \\
\text { Requirements }\end{array}$ & Not ES\&H \\
\hline RLPD 430.1 & Hanford Site Systems Engineering Policy & Not ES\&H \\
\hline RLPD 5000.1 & Site Management System & Not ES\&H \\
\hline $\begin{array}{l}\text { DOE/RL-92- } \\
49\end{array}$ & $\begin{array}{l}\text { Radiological Assistance Program Plan - } \\
\text { Region } 8\end{array}$ & $\begin{array}{l}\text { This order does not directly impact the safety of the } \\
\text { facilities. }\end{array}$ \\
\hline $\begin{array}{l}\text { DOE/RL-93- } \\
75\end{array}$ & Hanford Facility Contingency Plan & Implemented \\
\hline $\begin{array}{l}\text { DOE/RL-94- } \\
02\end{array}$ & Hanford Emergency Response Plan & Implemented \\
\hline $\begin{array}{l}\text { DOE/RL-92- } \\
36\end{array}$ & $\begin{array}{l}\text { Hanford Site Hoisting and Rigging } \\
\text { Manual }\end{array}$ & Implemented \\
\hline $\begin{array}{l}\text { DOE/RL-94- } \\
125\end{array}$ & Federal Building Self Protection Plan & $\begin{array}{l}\text { This order does not directly impact the safety of the } \\
\text { facilities. }\end{array}$ \\
\hline HSL\&T-1 & Hanford Site Lock and Tag Standard & Implemented \\
\hline $\begin{array}{l}\text { DOE/RL-94- } \\
97\end{array}$ & $\begin{array}{l}\text { Selection of Analytical Methods for } \\
\text { Mixed Waste Analysis at Hanford }\end{array}$ & $\begin{array}{l}\text { This order does not directly impact the safety of the } \\
\text { facilities. }\end{array}$ \\
\hline
\end{tabular}


HNF-6071, Rev. 0

\begin{tabular}{|l|l|l|}
\hline \multicolumn{1}{|c|}{ RL DIRECTIVES } \\
\hline $\begin{array}{c}\text { DOC. } \\
\text { NUMBER }\end{array}$ & \multicolumn{1}{|c|}{ TITLE } & \multicolumn{1}{|c|}{ BASIS } \\
\hline $\begin{array}{l}\text { DOE/RL-94- } \\
55\end{array}$ & Hanford Analytical Services QA Plan & $\begin{array}{l}\text { This order does not directly impact the safety of the } \\
\text { facilities. }\end{array}$ \\
\hline DOE-0223 & RL Emergency Implementing Procedures & Implemented \\
\hline DOE-0225 & $\begin{array}{l}\text { Hanford Emergency Assessment Resource } \\
\text { Implemented }\end{array}$ & \\
\hline
\end{tabular}

